\title{
COORDINATION OF ALLOCATION MEASUREMENTS IN OFDM BASED AD HOC OVERLAY SYSTEMS
}

\author{
Ulrich Berthold, Holger Heimpel, Friedrich K. Jondral \\ Institut für Nachrichtentechnik, Universität Karlsruhe, Germany \\ Email: berthold@int.uni-karlsruhe.de
}

\begin{abstract}
With an increasing demand for mobile communications an efficient usage of the available spectral resources gains importance, since suitable frequency bands are limited. Although nearly all frequency bands are assigned to specified services and users causing a frequency shortage, measurements show that nevertheless there are a lot of unused spectrum holes in the time frequency domain, leading to an overall low spectral efficiency [1]. In this paper we consider an overlay scenario with an OFDM (orthogonal frequency division multiple access) based additional ad hoc overlay system operating in the same frequency band as the licensed system and exploiting the spectrum holes. Since spectrum sensing is a key issue for the coexistence of both systems we propose a method in the MAC (medium access control) layer for frame synchronization, enabling the coordination of allocation measurements. Furthermore, we point out some signal processing aspects regarding overlay systems.
\end{abstract}

Index Terms - radio ressource management, OFDM, spectral efficiency, overlay systems, ad hoc networks

\section{INTRODUCTION}

With an increasing demand for mobile communications an efficient usage of the available spectral resources gains importance. Due to the static assignment of frequency bands to specific users by regulatory bodies, often the current communication demand does not match the available bandwidth. Two situations can occur: Either a user has more bandwidth available than he can use, resulting in spectral inefficiency. Or the demand for communication can be temporarily higher than the assigned bandwidth can provide, leading to a service degradation. One approach to overcome these static frequency assignments and to dynamically adapt the available bandwidth to different systems is the concept of spectrum pooling [2]. In an assigned frequency band the licensed system has highest priority and operates as usual. Additionally, an overlay system is deployed in the same frequency band. This secondary

The authors gratefully acknowledge that their work on Techniques, Algorithms and Concepts for Systems Coexisting with Licensed Systems in the Same Frequency Band (TAKOKO) is supported within the priority program No. 1163 (TakeOFDM) by the German Research Foundation (DFG).

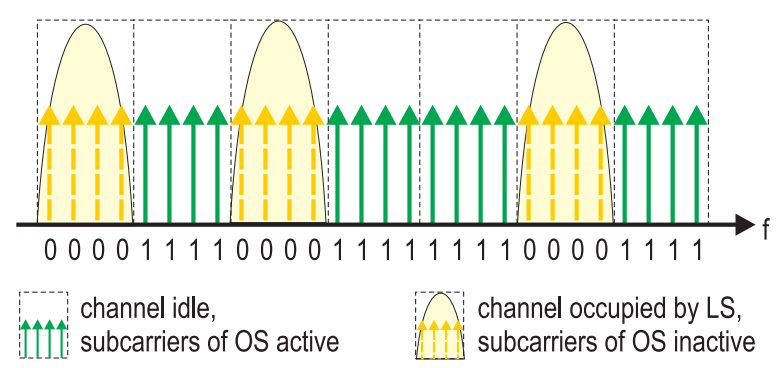

Fig. 1. Access of the licensed system (LS) and activated subcarriers of the overlay system (OS).

system only must use the free spectral resources not needed by the licensed system, leading to a significant impact on the design of the MAC layer as well as the physical layer of the overlay system.

The remainder of this paper is organized as follows. In section II the assumed overlay scenario is discussed in detail. We propose a new AHO (ad hoc overlay)-MAC protocol, enabling the coordination of spectrum measurements in an ad hoc overlay system in section III. Simulation results are discussed in section IV, followed by some signal processing aspects in overlay systems in section V. Finally this paper is concluded in section VI.

\section{AD HOC OVERLAY SCENARIO}

In this paper we consider an overlay scenario consisting of a licensed system and an overlay system which are operating independently [3]. Both systems are coexisting in the same frequency band. Since the licensed system neither must be modified nor notice the existence of the overlay system, we assume that there is no inter-system communication available. This directly implies that the overlay system has to derive the available resources by taking measurements of the spectral density and thus detecting the allocation of the licensed system. Due to the time variant property of the licensed system the overlay system has to perform the measurements periodically. Furthermore, we assume in our overlay scenario that the licensed system uses a multiple access scheme based on FDMA (frequency division multiple access) or TDMA (time division 


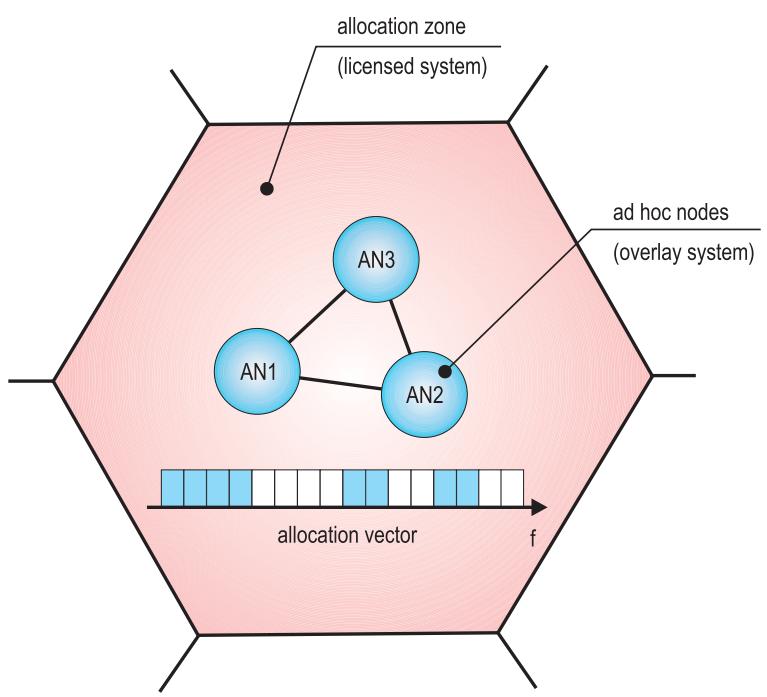

Fig. 2. Overlay scenario with ad hoc overlay nodes and a licensed system with one allocation zone.

multiple access) or a combination of both. CSMA/CA (carrier sensing multiple access with collision avoidance) is not a suitable access scheme for our scenario. Applying CSMA/CA, the licensed system would change its behavior in presence of an overlay system due to its sensing property, thus resulting in a decreasing performance in the licensed system. For the overlay system we use OFDM, since it embodies a sophisticated, flexible and efficient technology [4]. The FFT needed for the inverse OFDM operation can also be used for the allocation measurements, reducing hardware costs. In the overlay system the given frequency band is subdivided into several subcarriers the number of which is depending on the FFT size. Thus, the result of a measurement is an allocation vector, in which each binary element represents one subcarrier, indicating whether or not it may be used for overlay transmissions. This allows for a dynamic and efficient exploitation of spectrum holes left by the licensed system in the time frequency domain, since each subcarrier can be turned on or off individually even on a symbol basis, depending on the allocation vector. Figure 1 depicts the allocated channels by the licensed system at a certain time with the activated subcarriers of the overlay system filling the resulting gaps. Figure 2 finally visualizes the assumed scenario where the licensed system has one allocation zone with a time varying but location independent allocation vector. In this allocation zone there are several overlay nodes which are assumed to be operating in ad hoc mode.

\section{COORDINATION OF ALLOCATION MEASUREMENTS}

An overlay node in ad hoc mode has to meet two main challenges in order to establish communication links to other over-

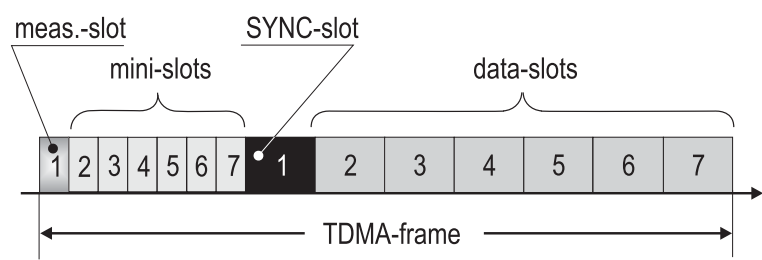

Fig. 3. TDMA frame with mini slots and data slots.

lay nodes. As in every overlay scenario it firstly has to consider information about the licensed system's allocation in order to avoid interference. Additionally, due to the ad hoc properties of the overlay system, the node needs information about other overlay nodes existing in the neighborhood. In this section we present an approach for the MAC layer which considers both aspects.

\subsection{MAC Frame Structure}

Many common MAC protocols for ad hoc systems are based on CSMA/CA techniques, e.g. DCF (distributed coordination function) etc. Nevertheless, in the context of an overlay scenario this approach is not suitable due to it's sensing property. In order to detect the licensed system's access, all overlay nodes have to pause their transmissions and perform the measurement at the same time. Otherwise overlay nodes could not distinguish between the licensed system's access and other ongoing transmissions in the overlay system. The coordination of the measurement phases is an important task of the MAC layer. In a centralized architecture the base station could easily resume this function, but in ad hoc mode this problem has to be solved differently. Here the overlay system needs to have self organization capabilities.

Since CSMA/CA is an unsuitable technique in this situation, we will focus on another class of MAC protocols: synchronized protocols based on TDMA. In [5] a protocol called multiservices dynamic reservation (MDR) TDMA is proposed with the purpose to serve as a general MAC framework combining CBR (constant bit rate), VBR (variable bit rate) and packet data. Similar to PRMA (packet reservation multiple access) a TDMA frame is divided into two blocks of slots: The first block contains a set of mini slots whereas the second part has the same amount of data slots.

In our proposed ad hoc overlay (AHO-) MAC protocol we adopt the concept of mini slots and data slots. Figure 3 shows the applied general frame structure. Two slots are reserved for special purposes. The measurement of the licensed system's allocation is performed in the first mini slot. Since each mini slot has a corresponding data slot, the first data slot cannot be used for data transmission. It therefore is used for synchronization tasks, i.e. detection of neighboring nodes and frame synchronization. The remaining slots are available for data transmissions. In general the number of data and mini slots can be arbitrary, depending on the required measurement in- 
terval.

\subsection{Frame Synchronization and Node Detection}

A new overlay node entering the scenario at first has neither information about the available subcarriers nor about other overlay nodes nearby. Thus, the overlay node always starts operating in scanning mode and performs the following steps in order to retrieve the necessary information:

1. In the first step it takes a measurement of the spectrum allocation in order to derive the current allocation vector. This can only be a first estimate, since the coordination with other overlay nodes has not been performed until then. Furthermore, it listens for synchronization messages from other overlay nodes.

2. In case the node cannot detect any synchronization messages within a defined time, it assumes that there are no other overlay nodes nearby and starts sending synchronization messages itself during the SYNC slot. During times when the node is not transmitting, it listens for synchronization messages sent by other overlay nodes nearby. Only subcarriers in channels that are not allocated by the licensed system are used.

3. If the node detects a synchronization message it begins frame synchronization and switches to association mode.

After performing these steps, at least two nodes form a synchronized cluster in which allocation measurements can be taken and data connections set up. Since there is no difference between the nodes (e.g. master / slave assignment), each overlay node has to follow the same set of rules. Thus, each overlay node always tries to send a synchronization message during the SYNC slot, even if a node already received a synchronization message in the same SYNC slot. The message is only sent when currently no other message is detected. The advantage is, that new nodes entering the edge of the cluster will recognize and can synchronize to it, thus increasing its size. Note, that several synchronization messages fit into one SYNC slot and that each message is sent with a random offset within the SYNC slot in order to decrease the probability of collisions. This offset is included in each synchronization message. From all received synchronization messages the one that was sent earliest is chosen for a frame re-synchronization, all others are only used for the detection of nearby nodes.

\subsection{Data Transmission Coordination}

We now describe the main steps for creating a data connection. It is assumed that frame synchronization was already performed successfully, only the available subcarriers are used and the node has knowledge about other overlay nodes within

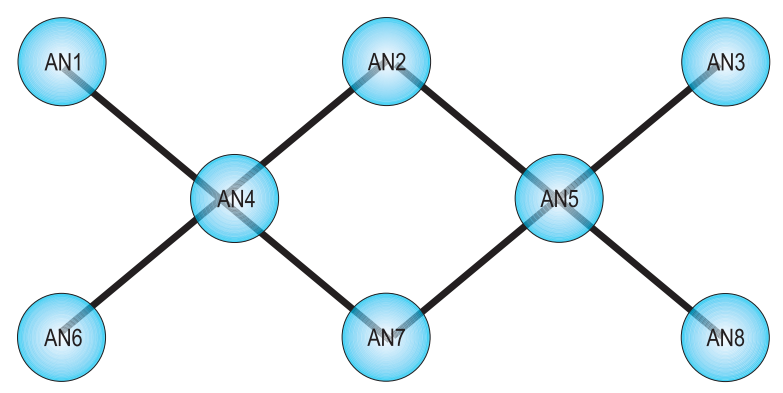

Fig. 4. Ad hoc network constellation used for simulations.

transmission range. In order to setup a connection to a neighboring node, the initiative node performs following actions, which are similar to reservation-TDMA:

1. By observing the mini slots and the corresponding data slots the node recognizes ongoing transmissions in the overlay system and determines available slots, one of which is chosen at random.

2. Similar to CSMA/CA a RTS (request-to-send) is sent within the chosen data slot, delayed by a random offset. The upper bound of the offset is determined in a way that the RTS always arrives completely within the data slot. During the offset the node is listening whether or not another node already has sent a RTS. Collisions can occur when several nodes want to use the data slot and send a RTS at the same time. In this case the procedure is repeated in the next frame with a different offset. If a node still has not been successful it chooses a different data slot.

3. The receiver receives the RTS and answers with a CTS (clear-to-send) in the corresponding mini slot. The connection now is established and by observing the data and mini slots all other nodes within transmission range of both communicating nodes are notified about the current transmission. Thus, this method avoids hidden nodes. The number of successive frames for the established connection can be limited in order to provide fairness among all concerned nodes.

\section{SIMULATION RESULTS}

For simulations the AHO-MAC protocol has been implemented with a discrete event simulation tool. We especially investigated the frame synchronization and node detection parts of the protocol. Therefore we used the node constellation as depicted in Figure 4 with a grid containing eight overlay nodes. Nodes that in general have the possibility to communicate due to their distance are connected with a line. Each node is activated with a random offset, leading to an initially unsynchronized state between the nodes. The used frame consists of 
three data slots and three mini slots. Including the measurement and SYNC slot this leads to a frame length of $480 \mu \mathrm{s}$ when one data slot has $100 \mu \mathrm{s}$ and a mini slot $20 \mu \mathrm{s}$. Simulations show that a synchronized network can be achieved within only a few frames: 75 percent of the nodes form a synchronized cluster after two frame periods, all nodes are integrated in the cluster after four frames. Further investigations are being done, especially with more general and larger networks in order to get a more general statement for the performance of the protocol.

\section{SIGNAL PROCESSING ISSUES}

Regarding signal processing there are several important aspects that need to be considered in OFDM based overlay systems. In fact, the allocation vector is an overlay specific parameter that inherently affects the whole system design, especially the physical and the MAC layer. For this reason a crosslayer approach is unavoidable [6].

Since the goal of the AHO-MAC protocol is the coordination of allocation measurements in order to determine which subcarriers may be used, detection plays a vital role in this context. Based on measurements given by the FFT, the actual allocation vector has to be derived. Here the NeymanPearson criterion, known from radar applications, can be applied, which maximizes the detection probability at a given false alarm probability. Better performance can be achieved when applying a distributed detection approach, exploiting diversity [7]. Thus, regarding ad hoc networks, the detection gets more accurate after several nodes have formed a cluster and have exchanged local measurement results.

Another for the AHO-MAC protocol relevant issue currently being investigated is frame synchronization. When designing the preambles two aspects have to be considered. First, as in every system the used preambles must have certain properties that allow an optimum detection of the beginning of the frame. Second, the interference caused by the preamble on the subcarriers used by the licensed system must be minimized. Since the available subcarrier configuration can change dynamically, synchronization messages in the SYNC slot must use adaptive preambles depending on the current allocation vector in order to minimize the interference, simultaneously ensuring a good frame detection.

The reduction of out-of-band radiation is in general an important issue for overlay systems, since the performance of the licensed system must not be affected. A promising approach is the insertion of cancellation carriers, which are calculated depending on the current OFDM-Symbol, in order to compensate the occurring interference [8].

\section{CONCLUSION}

In this paper we considered an overlay scenario including a licensed system and an overlay system coexisting in the same frequency band. The overlay system is using OFDM and operating in ad hoc mode. In order to coordinate the allocation measurements within the overlay system we proposed a new MAC protocol called AHO-MAC, enabling a frame synchronization and node detection for the overlay ad hoc nodes. Simulations show, that nodes are synchronized within a maximum of four frames. Furthermore, we pointed out some signal processing aspects important for the AHO-MAC protocol, especially distributed detection and synchronization.

\section{REFERENCES}

[1] M. A. McHenry, P. A. Tenhula, D. McCloskey, D. Roberson, and C. Wood, "Chicago spectrum occupancy measurements \& analysis and a long-term proposal," in Proceedings of the First International Workshop on Technology and Policy for Accessing Spectrum (TAPAS). August 2006, www.wtapas.org.

[2] J. Mitola, "Cognitive radio for flexible mobile multimedia communications," in IEEE International Workshop on Mobile Multimedia Communications, San Diego, CA, USA, November 1999, pp. 3-10.

[3] U. Berthold, S. Brandes, M. Schnell, and F. K. Jondral, "On Focus: OFDM Based Overlay Scenarios," in Proceedings of the 2006 IST Mobile and Wireless Communications Summit, Myconos, Greece, June 2006, CDROM.

[4] T. Weiss and F. K. Jondral, "Spectrum Pooling - An Innovatiove Strategy for the Enhancement of Spectrum Efficiency," IEEE Communications Magazine, vol. 42, no. 3, pp. S8-S14, March 2004.

[5] D. Raychaudhuri and N. D. Wilson, "ATM-Based Transport Architecture for Multiservices Wireless Personal Communication Networks," IEEE Journal on Selected Areas in Communications, vol. 12, pp. 1401 - 1414, October 1994.

[6] U. Berthold, S. Brandes, M. Schnell, and F. K. Jondral, "A framework for crosslayer optimization in OFDM based overlay systems," in Proceedings of the 11th International OFDM-Workshop, Hamburg, Germany, August 2006, pp. $253-257$.

[7] T. Weiss, J. Hillenbrand, and F. K. Jondral, "A diversity approach for the detection of idle spectral resources in spectrum pooling systems," in 48. Internationales Wissenschaftliches Kolloquium, Ilmenau, September 2003, CDROM.

[8] S. Brandes, I. Cosovic, and M. Schnell, "Reduction of Out-of-Band Radiation in OFDM Systems by Insertion of Cancellation Carriers,' IEEE Commun. Lett., vol. 10, no. 6 , pp. 420-422, 2006 